Peer-Reviewed Article

ISSN: 2162-3104 Print/ ISSN: 2166-3750 Online

Volume 6, Issue 1 (2016), pp. 107-127

(C) Journal of International Students

http://jistudents.org/

\title{
Loyal Tongue, Liberal Mind: International Students' Experiences on Dietary Acculturation in England
}

\author{
Novella O'Sullivan \\ Liverpool Hope University (UK) \\ Farzad Amirabdollahian \\ Liverpool Hope University (UK)
}

\begin{abstract}
This study explores the dietary experiences of international students in a British university, and how these phenomena differ from what they experienced in their home country. Ten participants were recruited using purposive sampling. The inclusion criteria were international students who had lived in England for less than a year; those with diet-related health problems were excluded. Data were collected using semi-structured interviews and analyzed with Colaizzi's method. This resulted in three main themes: changes in diet, new dietary experiences, and factors affecting dietary practices. Our findings also offer new insight about what international students might encounter relating to food and diet when studying abroad.
\end{abstract}

Keywords: acculturation, diet, eating habit, food choice, foreign student, phenomenology

The number of international students engaged in higher education has been increasing during the last decade; the United Nations Educational, Scientific and Cultural Organization reported a $12 \%$ rise of international students number annually (Coughlan, 2011). Anglophone universities are the most popular destination, such as the United States (US), the United Kingdom (UK), Australia, and Canada (Ryan, 2011; Verbik \& Lasanowski, 2007). 
International students help provide a global experience for the universities, in addition to their contribution to the host country economy (Ramachandran, 2011). The UK, as the focus of this study and one of the most visited countries, have benefited from the overseas students. During 2011-12, data show that international (European and non-European) students in the UK generated up to $£ 3.6$ billion in tuition fees and $£ 7.37$ billion in off-campus expenditure (Universities UK, 2014). Furthermore, their presence in the classroom means that both the home students and international students can share perspectives and learn through the experiences of others who come from different backgrounds (Ramachandran, 2011).

However, recent policies that implemented stricter rules on UK visa application (Morgan \& Matthews, 2014) might have caused a decrease in number of international students at British universities. The Higher Education Founding Council for England (2014) considered this first decline in past three decades as a warning for their higher education institutions to put more effort in attracting foreign learners.

As a result, British universities are under pressure to improve not only their quality of teaching and learning, but also the international students' health and wellbeing. There has been more awareness for the later, particularly since health and wellbeing are considered as part of the factors influencing academic performance (Ansari \& Stock, 2010). A relevant aspect that can be observed is the students' dietary health. Indeed, changes in diet have long been reported as a welfare issue for international students worldwide (Edwards, Hartwell, \& Brown, 2010; Hannigan, 2007; Macrae, 1997).

Taking these matters into account, we conducted a primary study that aimed at understanding wellbeing of international students in the UK. More specifically, we investigated their dietary experiences, including eating habits and the factors that might influence their diet when living away from their home country. Comprehending these issues from the international students' perspectives might help higher education institutions in their attempt to improve students' wellbeing and satisfaction rate.

\section{LITERATURE REVIEW}

During their sojourn in attaining higher education abroad, international students may be exposed to cultural differences that result in acculturation. One of the changes that students have to cope with is a different food culture, which may lead to changes in dietary habits (Satia-Abouta, 2003). These changes may have health consequences such as obesity and chronic diseases (Gilbert \& Khokhar, 2008; Papadaki \& Scott, 2002). 
Nevertheless, recent research is limited regarding the food habits and dietary health of international students when studying abroad (Hartwell, Edwards, \& Brown, 2011). Most studies have indicated that consumption of fruit and vegetable decreased (Alakaam, Castellanos, Bodzio, \& Harrison, 2015; Edwards, Hartwell, \& Brown, 2010; Kremmyda, Papadaki, Hondros, Kapsokefalou, \& Scott, 2008; Perez-Cueto, Verbeke, Lachat, \& Remaut De Winter, 2008; Reeves \& Henry, 2000). Increased consumption of fast food and sugary snacks were also reported (Alakaam et al., 2015; Cappellini \& Yen, 2013; Kremmyda et al., 2008). International students were aware of the bad effects of fast food and confectionery consumption; some revealed that they felt guilty about this (Alakaam et al., 2015). However, as far as most international students were concerned, it was part of an adjustment process to the new food culture (Brown, Edwards, \& Hartwell, 2010; Cappellini \& Yen, 2013).

Past research trend on the diet of international students in western countries have been related to weight gain - several studies suggested that transition to the new culture involves a higher intake of western food as part of dietary change and hence increases the body mass index (Abraido-Lanza, Chao, \& Florez, 2005; Gordon-Larsen, Harris, Ward, \& Popkin, 2003; Lara, Gamboa, Kahramanian, Morales, \& Bautista, 2005; McDonald \& Kennedy, 2005). On the other hand, several researchers have found in their qualitative studies that international students became aware of gaining weight and tried to control their energy intake (Brown, Edwards, \& Hartwell, 2010; Hartwell, Edwards, \& Brown, 2011).

We propose that changes in an anthropometric measure (e.g. body weight) alone cannot fully reflect how healthy a diet is. For example, skipping meals -particularly breakfast- was common among those who managed to maintain their body weight (Edwards, Hartwell, \& Brown, 2010; Pan, Dixon, Himburg, \& Huffman, 1999; Reeves \& Henry, 2000). This justified the need to look more into the food choices and eating habits of international students to understand their dietary health, rather than simply measuring the body mass index.

\section{RESEARCH METHOD}

This study used a qualitative approach due to its ability in providing indepth understanding of a phenomenon. Participants' lived experiences were gathered to construct a shared meaning, according to the nature of phenomenology (Moustakas, 1994).

The study was reviewed by the Health and Social Science Ethics Committee of Liverpool Hope University. After obtaining ethical approval, ten participants were recruited using purposive sampling, so as to access 
only knowledgeable people who could give relevant information to the research. The inclusion criteria were international students in England who had been living in England for less than a year - in order to collect valid data and avoid memory bias. Exclusion criteria were the occurrence of eating disorder and/or any diet-related health problems, hence the participants would be those with individual free choice in the food consumed.

Semi-structured interviews were conducted; the open-ended questions gave freedom to the participants to express their opinions and perceptions pertinent to the topic. The topic guide covered general questions regarding the participants' daily eating pattern, changes in eating habits (if any), food choices, and factors affecting them. Background information was given to the participants before any data were collected, and they were asked to sign the consent form. The entire interview sessions were audio-recorded to aid verbatim data transcription.

Data collection was set in a university in the North West of England. A closed and quiet room with sufficient lighting was chosen as the study setting to provide a comfortable environment for the participants. A pilot study was conducted to inform us if there was any issue on the content and language of the questions, and predict how long it would take to complete an interview session. After the pilot study, several modifications on word selection and question sequence were made.

\section{Participants}

Participants' profiles are listed below; instead of using names, they will be addressed with their self-declared nationality in this study:

1. Sri Lankan student, female, age 26, single.

2. Lebanese student, female, age 29, married.

3. Maltese student, female, age 20, single.

4. South Korean student, female, age 25, single.

5. Dutch student, female, age 22, single.

6. Mauritian student, male, age 25, single.

7. Romanian student, female, age 21, single.

8. Ghanaian student, male, age 45, married.

9. Bangladeshi student, female, age 26, married.

10. Irish student, female, age 23, single.

Except the Ghanaian student who lived with his wife and children at his home country, all other participants lived with their parents at home before they came to the UK.

The interview sessions for each participant lasted for around 45 minutes. Data collected were analyzed manually to familiarize us with the materials. We employed a descriptive analysis based on Colaizzi's (1978) method. The 
'bracketing' feature in Colaizzi's method allows researchers to set aside their preconceptions of the matter (Walsh, 1988). Thus, the research result would reflect the participants' perspectives with minimal bias, showing the real nature of the phenomenon in a study (Shosha, 2012).

The first step of data analysis was to read through all of the transcribed data. Significant statements were extracted from each participant's data. There were 392 significant statements derived from ten interview protocols. The next step was to formulate meaning out of each statement. Meanings that were similar or related to each other were assembled into a category or cluster of themes. The data analysis generated twelve theme clusters. These were then grouped together according to their distinctive features, which formed the three main themes. It is worth noting that during the analysis of the last participant's data, there was no new theme emerged, signifying that data saturation had been reached.

\section{RESULTS}

Three themes emerged from the data analysis process, which involves the changes in the participants' diet, their new experiences relating to food, and the factors that might have affected their dietary practices.

\section{First theme: Changes in diet}

During their study in England, the international students in this study reported changes in their eating habit, the type, and amount of certain food consumptions, such as fruit and vegetable, as well as fast food. Some participants also suggested that their new diet had caused weight change.

Irregularity in eating pattern. Almost all participants admitted that they had more regular eating patterns at home. This changed after living in England, characterized by the increased frequency of skipping meals.

As I mentioned before, I started skipping lunch. I noticed that I started skipping breakfast as well. At home, even at the weekend we'd have like, a regular eating pattern. Whereas here, it's just either or, could be nothing until the evening and then one big meal or something small at lunch time. (Irish student)

Only one participant regarded his eating pattern to be more regular in England. This was due to his profession as a priest and the factor of food availability and accessibility: 
This time around I eat more regularly than I was in Ghana. And uh, mostly I have something to eat here. And here too, I am almost every time indoors, except that when I'm in university, or I'm out for program in church. So I'm closer to the food, or fruits. So the eating habit here is actually far better compared to in Ghana. (Ghanaian student)

The reasons that contribute to this irregular eating pattern are discussed further in the factors that affect the dietary practices (the third theme of this study).

Fruit and vegetable consumption. When participants were asked about the biggest difference in their eating habits since moving to England, half of them immediately referred to "less fruits and vegetables".

The big difference is that I don't really eat a lot of fruits and vegetables, and at home I eat a lot of them. . . It's always very balanced. Here if I make, um, sausage or something, I'll just eat those or I'll eat them with onion rings or something. (Maltese student)

As experienced by this student, almost all other participants stated that they used to consume adequate amount of fruit and vegetable at home due to family influence. A Dutch student explained that at her home, "there will always be veggies . . . we basically eat every dinner with veggies." She admitted that the way she was raised later affected her eating habits in England. When shopping for food she would "first go to the fruits and veggies and milk section, and just buy that first". That way, she could maintain her fruit and vegetable consumption in sufficient quantities.

Unhealthy food consumption. Unhealthy food is defined as food containing high levels of fat, sugar or salt, which contribute to an unhealthy diet (Matthews, 2007). In this study, we also included confectioneries or sweets, convenience food or junk/fast food that is easy to purchase and consume (Ashakiran \& Deepthi, 2012).

Most participants admitted that they had consumed more junk food and confectioneries since living in England. The Sri Lankan student felt that, "I'm consuming lots of sugary foods after I come here." A Ghanaian participant added:

To be honest with you, every time I'm going to campus I rely so much on biscuits. Because I leave very early, I can't eat very 
early. Sometimes I don't want to walk out to a restaurant and eat anywhere, so I have to [rely on biscuits], I carry along some biscuits and eat that.

The Maltese student had her own story. She came to England without sufficient food preparation skills; as a result, she skipped meals more frequently, or opt for junk food:

I get on by a lot of things like the freezer food and things like that. I eat a lot of things like, uh, chicken nuggets or onion rings, or like potato wedges, things that you just take out of the freezer and stick in the oven. I order a lot of pizza with one of my friends, and I make pasta . . . and that's about it: freezer foods, pasta, and pizza.

Some participants agreed that the portion size of fast food purchased affected the amount they consumed - for example, the South Korean student indicated that

Because I love the pizzas, so I had pizza like two times a week; that could be so messy. Because the pizza is so big like this \{depicting the size of big pizza with hand, and then you eat by yourself. Then you cannot finish them all, so you froze that pizza and then eat the pizza again the next meal or next day. So it's like, you're having a lot of calories.

However, some students were pleased with that situation. The Maltese student mentioned that along with her peers, they purposely ordered bigger portion and saved the leftovers for their next meal. "I mean, I got away from cooking less!" she exclaimed.

Beside peer influence, the reasons behind junk food consumption include the convenience factor. As the Irish student remarked, "It is a lot easier just to go for like a convenient meal or canned food, or, you know like a pizza [that] you can just put in the oven".

Weight change. Half of the participants reported that they experienced weight change. Two participants suggested that they gained weight; the other three reported weight loss.

[I gained] maybe 5 kilos ... In England, I chose unhealthy foods. And then I do regret that a lot, so I decided not to eat pizza and chips anymore. (South Korean student)

[My weight] decreased by two or three kilos, because I didn't eat properly, I didn't eat when I [wanted] to eat, I [did not] like the food, I was completely stressed. (Romanian student) 
From their descriptions, it can be concluded that the participants tend to link their weight changes to eating habits in comparison with previous habits at home, the types of food consumed, eating patterns, and its healthiness.

\section{Second theme: New dietary experiences}

This theme describes how the international students in our study had the chance to try new food, experienced different kind of food culture, and expressed their opinion or preference in food and diet after living in England for several months.

Preference for traditional food. In addition to its cultural aspect, traditional food can be appreciated for its health potentials. Trichopoulou, Soukara, and Vasilopoulou (2007) reported that tradition tended to honor food that was both palatable and healthy. This was consistent with reports from participants who thought their traditional home-cooked as healthy "real meals". The Lebanese student suggested that, "the Mediterranean meals are healthy. They include lots of vegetables, we eat lots of fruits." Likewise, the South Korean student said, "If you just follow the [traditional] Korean food, then you hardly gain weight, you hardly gain diseases."

Some participants looked for traditional food in England or brought traditional spices from home to help them provide a familiar taste in England. International students tended to find students from their home country and have traditional food together, especially on weekends or holidays. However, they would also sample global food provided by their peers:

Sometimes, for example, with American thanksgiving, we celebrate it like all of us, all the international students. So we had a meal with turkey and stuff, which I've never had before in that sense. So in that case, yeah, you'll eat different stuff. (Dutch student)

Initiated by the university, they would sometimes organize a 'bring-a-dish' party - a chance to gather with fellow international students and taste traditional dishes from other countries.

Perception of the "English experience". This category covers participants' feelings and reflection on their dietary experiences in England, as well as the "English food" or food common to the English culture.

Almost all participants regarded the experience of living in England as a positive thing, which allowed them to learn how to prepare food and to 
try new food. Besides new tastes, participants also appreciated the control over their own food choices:

I enjoy it. I enjoy cooking. I enjoy eating here; I enjoy selecting those types of foods that I like. Um, wish I have more variety, more choices to choose from, but . . . it was a very positive experience. (Lebanese student)

Despite the positive experiences of enhanced food selection and food preparation skills, the food commonly found in the English culture was considered as less appetizing, unhealthy and did not fulfill the international students' taste. The Mauritian student said that "the British food... I don't know, I mean, it's kind of bland. They don't put much sauce or spices, you know." The Romanian student agreed:

I feel like it doesn't have a taste at all. I don't know, it tastes different ... Actually I'm not the only one who said that, you know. Because there are a lot of people that are saying they're not happy with English food.

All participants admitted that they would go back to their previous eating habits at home when leaving England because they had been used to it and considered it healthier.

Well I think if I go back to Mauritius, I think things will go back to normal, back to my eating habit. Because my mom, my family, they have their own way of eating. I was eating the same stuff; they're cooking for me. (Mauritian student)

One participant admitted that she had already become accustomed to her eating habits in England, including "bad habits" such as skipping meals, and would find it hard to switch back.

I hope that I can get back to my previous eating habit. But I know I'm [going to] find it hard. I've kind of settled with my ways now. Because I've never really had to look after myself before. And now that I've started to, I know I've got into some really bad habit. (Irish participant)

In summary, this theme demonstrates how the international students might perceive food acculturation as a positive experience. However, as 
indicated by our participants, they would prefer home taste and their previous eating habits before coming to England.

\section{Third theme: Factors affecting dietary practices}

This section shows how the participants contemplated the factors that had affected their dietary practices while they were studying overseas.

Health concerns. Concerns about being healthy and/or consumption of healthy food have been repeatedly mentioned as underlying reason for changes in dietary habits.

All participants considered their eating habits at home as healthy. Food was prepared by parent(s) or other family members, ensuring regular meals. This was illustrated by one participant: "In the morning, like, my mom would make me a boiled egg or something . . . Here [in England] I never eat breakfast."

Another factor was that the food selections at home were perceived to contain more useful nutrients. The Romanian student observed that her eating habit at home was "healthy . . . Because the food is not [fattening], and um, it contains vitamins and proteins and everything the body needs."

Eating habits in England was considered less nourishing than at home for most of the participants. They were aware of it, yet unsuccessful in maintaining the ideal eating habit, due to factors discussed in this paper. The Sri Lankan student was worried that "in long term, I will definitely have problems, I will definitely have problems [due to current unhealthy eating habit]."

Nevertheless, health concerns were considered as part of the factors affecting diet at home and in England. The Mauritian student reflected, "It was very important to have healthy food, you need to have a very good balanced diet”. Regarding his dietary practice in England, the Ghanaian student described that, "the factors are also health. I try to eat something that will keep me going, because I don't want to fall sick. I think basically that it is. Because it's my health that I try to protect."

Individual determinants. This category includes the participants' personal taste, mood, and beliefs or religious factors related to their dietary habits. Personal taste affected food selection in both the participants' home countries and in England. Some international students found it hard to adapt to the taste of the "English food", which was considered less tasty compared to the food they consumed at home. For example, the Romanian student believed that the meat in England “doesn't have the same taste". The Sri Lankan student also expressed her opinion: 
The fruit that I can find here is a little different than in my country. I don't like that taste much . . . Back in my country, the type of green leaves and here, [the] green leaves, [they are] totally different, I can't adapt to them. I mean sometimes I feel like, how I can eat this kind of leaves?

On the other hand, mood as well as stressful conditions could affect the appetite for some participants.

If you're in a good mood, you will feel [like wanting] to eat. If you're in a bad mood, you won't ... [Being] stressed will make you eat less. For example, when I'm having exams, I always eat less. (Bangladeshi student)

Religious factors could also influence eating habit in term of food selection, where the international students would avoid certain types of meat. A Muslim participant stated clearly that, "my religion affects my [food] choices, yeah."

Influence of family and peers. The participants' food choices and eating habits were affected by their family and peers, both in their home country and in England.

Parental influence was as an important factor in determining healthy diet at home. As admitted by the South-Korean student, "Because my mom cooks, so we have to eat what she cooks". Parent(s) would prepare food that was considered nutritious by the participants and keep their eating patterns regular. The Dutch student described the difference between having family influence at home and not having one in England, "Because back home you have, like, people you live with that will wake you up, [saying] 'have some tea and breakfast' or whatever. Here, you just sleep, and no one bothers you."

In England, participants were living away from their relatives who would have reminded them not to skip meals or to maintain good quality food selections. Nonetheless, many participants figured that how their family raising them affected their eating habit even when they lived far from home. For instance, the Dutch student suggested that eating healthily in England "just comes naturally because I'm kind of raised that way".

Beside the family influence, peers also affected the participants' eating habit. Seeing friends during leisure time encouraged eating commercially prepared food such as junk food, both at home and in England. 
When you live with people your age, sometimes someone comes up to you and says like 'do you want pizza because I feel like having pizza', or like 'hey, we're going out, do you want some booze, I have some booze'. So in a way it could be like... yeah it was kind of like more unhealthy in a way. (Dutch student)

Living in halls and meeting other international students introduced the participants to new tastes. Some participants also learned how to make new dishes. The Lebanese participant shared this experience: "One of my flat mate used to make wraps. And I found that it's easy and healthy. I didn't make wraps back home. It was something that I learned here".

Food determinants. The food determinants that affected participants' dietary practices include food availability, price, storage (in terms of food's shelf life and space to store food), as well as food preparation issue.

Almost all participants would purchase the types of food available in local stores accessible to them: "Here [in England] I don't have time to worry about my food, therefore like I'm going for whatever available" and cook "whatever there is of course in the fridge". This affected their food choice and eating habits.

Some participants were satisfied by better food availability in England compared to their home country. For instance, a participant was pleased with the availability of free-range eggs in England, which she could not find in Korea. However, there were some types of food that were difficult to find in England, which caused decreased consumption. For example, the Lebanese and Ghanaian student found it hard to purchase fresh fish.

Price had a big influence towards international students' food selections and eating habits. Most of the participants included "price" or "budget" when discussing factors affecting their food choice in England; "first is health, second is budget".

Price could have a double-sided effect in determining health aspect in participants' eating habits. Those who considered eating out expensive chose to prepare food themselves. They reflected that "it was healthier than back home because no fast foods here, no junk foods". Some participants replaced certain foods that they perceived as expensive with cheaper options, or tried to find other stores with more reasonable price.

On the other hand, some participants considered fresh groceries more costly than convenient or frozen food. Hence, they would prefer the later. 
[The] frozen foods, those are so cheap! And other healthy things like mushrooms and vegetables, they are so expensive, I mean compared to the frozen foods. So, if you spend a lot of money, and you don't have much money right now then... then I choose some cheap things . . . Then anyway, you don't have to cook, and it's cheap, and it really makes you feel full. (South Korean student)

Apart from price, short shelf life of fresh food could discourage participants from purchasing them.

[The frequency of buying] veggies, not so much. Because I think it's difficult to store vegetables here ... you have to eat it off in two or three days ... so I think it did affect my eating habit. I was eating less vegetables. (Mauritian student)

Additionally, spaces to store the food also affected food choice. As remarked by the Irish student, "I have one freezer drawer and one little shelf in the fridge. So I suppose my choices are dictated by what I have room for."

Regarding food preparation issue, almost all participants were not used to preparing food for themselves at home. Food was provided by parent(s) who ensured balanced diet and regular eating patterns. Lack of cooking skills caused some participants to provide only simple dishes, or opt for convenience foods such as frozen food and junk food. In some cases it even caused participants to skip meals.

Some participants managed to maintain good eating habits by preparing food on their own in England. This was due to the satisfaction of choosing own meals and their fondness for cooking: "I really enjoy cooking and that's the main reason which I should say that influence my healthy diet". Some others saw improvements in their cooking skills after living in England and having to prepare food for themselves. The Maltese student reflected, "What's good is that I'm actually having to learn how to make things myself instead of just relying on everybody to cook for me".

Space in the kitchen for cooking was also a factor determining eating habits. Two participants admitted that they would avoid cooking in a busy kitchen, so that they would not be embarrassed about their lack of food preparation skills.

Having to share the kitchen . . . I don't like cooking in front of other people as well because I find it embarrassing cause I 
don't really know what I'm doing and I feel like they're just laughing at me \{laughs\}. (Maltese student)

Time Constraints. When time was available, participants could spend it cooking "real meals" properly, such as traditional food. On weekends, if participants had much more time and did not worry about study or academic schedules, eating patterns could be more regular.

Conversely, busy schedules on weekdays caused more skipped meals due to the limited time available for cooking. "The time is not enough for me to cook" and "[because] I didn't see that it worth the hassle" were popular opinions. This also encouraged unhealthful food consumption, as shown by their comments: "I just eat some snacks" and "If I don't have time, if I'm stuck with assignments, if I have to do something else, uh I'm just grabbing... pre-cooked [meals or] something".

Busy academic schedules and assignment matters were the main reasons for time restrictions. Despite the awareness of eating well, participants would prioritize their studying. Busy academic schedules discouraged participants from preparing food, because "when you study, and you have so many things to do, it's difficult to wake up early in the morning and prepare food for the day," explained the Mauritian student. When they were preoccupied with assignments, most participants would not cook. Some even skipped meals, as illustrated by the Irish student: "If I'm studying so I have a lot of work to do, I'll go to the library and spend all day in the library and not eat until the evening."

Environmental influences. Weather was a common theme that emerged among the participants, as an influence on their eating habit and appetite both at home and in England.

Yeah there are many factors [that affected food choice], um I guess, weather. You know when it's like gloomy weather or rainy seasons, I feel to take more like rich food. And like when it's hot . . . I preferred to take very light food. (Bangladeshi student)

For the participants, seasonal and geographical issue could influence food selections. Food choice at home depended on "what the farmers are planting". In England, the location of food stores close to international students living halls also affected the types of food purchased. 


\section{DISCUSSION AND CONCLUSIONS}

The study findings offer three important themes that addressed dietary experiences of international students living in England. Associations between themes are also evident. For instance, a participant mentioned that she did not have time to worry about food, so she would just opt for whatever was available in the nearest store. Such a situation links the time issue with food availability; altogether they may contribute to affecting food choice, as described in Shepherd's food choice model (Shepherd, 1985). This illustrates the numerous dimensions of factors affecting dietary practices, which contribute to the international students' dietary experiences.

Irregularity in eating pattern seen in our study was characterized by higher frequency of skipped meals when living in England. This adds to the previous findings that indicated breakfast as a meal commonly missed by international students (Edwards, Hartwell, \& Brown, 2010; Pan et al., 1999; Reeves \& Henry, 2000). While some participants in our study suggested that they would disregard breakfast in favor of a "lie-in" when they had an early class, we also found that skipping meals did not only involve breakfast. It could include any meal of the day depending on the reasons, such as financial issues, busy schedules, personal mood, or lack of space and privacy to cook in the kitchen.

Overall, fruit and vegetable consumption was reduced once the international students began residing in England. This has also been reported in studies by Reeves and Henry (2000), Kremmyda et al. (2008), PerezCueto et al. (2008), and Edwards, Hartwell, and Brown (2010). Our study clarifies that family influence is one of the main reasons behind this phenomenon. Almost all participants claimed that at home, their parents or other family members encouraged them to consume more fruit and vegetable, especially those who prepared food for them. Even those who managed to maintain healthy food consumption in England admitted that the habit of including fruit and vegetable in their diet remained because they were "raised that way" by their parents.

Junk food and confectionery consumption increased after the international students left their home countries. This is comparable to the findings by Cappellini and Yen (2013) and Kremmyda et al. (2008). Although Cappellini and Yen (2013) found that junk food acted as a bridge between participants and the local dishes, our study reveals that international students chose convenient food because it was an easy and affordable solution to avoid the cooking hassles. Furthermore, some participants in our study raised the issue of junk food portion sizes and the "leftovers" that led 
to increased consumption of this unhealthy food, overall giving further insight on the role of junk food in international students' dietary practices.

Regarding the implications of high intake of junk food for weight gain, several previous studies demonstrated that body mass index tended to increase during a transition into a new culture (Abraido-Lanza, Chao, \& Florez, 2005; Gordon-Larsen et al., 2003; Lara et al., 2005; McDonald \& Kennedy, 2005). Conversely, despite the increased consumption of junk food, only two out of ten participants in our study experienced weight gain. Three participants reported weight loss due to irregular eating patterns, decreased appetite caused by the unfamiliar taste of local food, and/or lack of cooking skills; represented by a participant who admitted that she relied on junk food to "get on by" and yet lost weight.

Hartwell, Edwards, and Brown (2011) indicated that international students tended to maintain their food culture, often by making traditional food on their own or eating together with fellow students from their home countries, sometimes using ingredients they brought from home. The same phenomena occurred with the participants of this study. While the qualitative study by Brown, Edwards, and Hartwell (2010) highlighted that taste was a factor prompting international students to seek familiar foods, our study stresses the importance of perception of healthy eating as a factor. Participants considered their home food more nutritious than the local food, and explained that by providing their own traditional food, they maintained a healthy dietary practice to "keep [them] going". This opinion is similar to that of the international students in Alakaam et al.’s (2015) study.

The international students in our study cherished having traditional food with their compatriots; in addition, they enjoyed sharing cultural food with students from other countries. Occasions such as "bring-a-dish" events where students could sample global dishes were mainly initiated via the host university. Yet, similar events were sometimes arranged by the international students themselves. This shows the shift of trend compared to a previous qualitative study concluding that "the drive to eat home country food implies the formation of mononational friendship groups, which may impede language and culture learning” (Brown, Edwards, \& Hartwell, 2010, p.206). Contrarily, our finding displays the participants' enthusiasm regarding experiencing new culture, friendship groups, and food.

Cappellini and Yen (2013) indicated that international students eventually returned to their home diet and they only considered eating local food as a temporary experience. Similarly, our participants also preferred to revert to their previous eating habits at home. Nevertheless, all of the participants regarded their eating experience in England as a positive one: some participants were pleased that they could have their own food choices in England, others had the chance to try or cook new dishes, and some had 
realized the importance of healthy food. Almost all of our participants improved their cooking skills during their stay in England. Many participants decided that these new skills would be implemented when they returned home.

There are several factors that influenced the dietary practices of our participants; a theme that often appeared during data analysis was health concerns. Most participants regarded their eating habits in England as less healthy compared to their habits at home country. This was based on food selections, which lacked fruit and vegetable and contained more junk food, as well as irregular eating patterns. Some participants addressed the "unhealthy English food" as a reason; international students in the study by Brown, Edwards, and Hartwell (2010) had a similar opinion about "English food" that was perceived as having high levels of fat and sugar.

International students' awareness of health and the consequences of unhealthy food consumption had also been reported in the studies by Brown, Edwards, and Hartwell (2010) and Cappellini and Yen (2013). In addition, we discovered that the participants were more concerned about their academic performance and outcome. Our findings highlight the role of academic issues, demonstrating how their study took priority compared with their diet. For instance, when the participants had to allocate a big portion of the time for university work, they would intentionally skip meals or refuse to cook. This is comparable to the findings of a similar research conducted in the US by Alakaam et al. (2015).

Our study helped provide an in-depth understanding of food choice determinants during dietary acculturation, including the availability of food, storage space, shelf life, and food preparation skills. Environmental issues such as weather and food seasons also affected appetite and food intake.

Overall, numerous factors that influenced international students' dietary practices in their home countries were comparable to those in England, with major difference in time constraints, food availability, and food accessibility. In England, international students had to make time to prepare food amidst their study. Meanwhile at home, there were family members who would provide food; hence time availability was not a major concern. Similarly, international students never had to worry about shelf life or storage issues in their home country.

Participants' backgrounds including nationality and occupation also contributed to their dietary experiences. There has been an assumption that Asian students are more resistant in adapting to Western food (Edwards, Hartwell, \& Brown, 2010). We found a pattern where participants from outside Europe were likely to seek traditional foods; however, Asian students in our study also did not find it difficult to adapt to local dishes or food from their peers' countries. 


\section{IMPLICATIONS}

Our study explores the experiences of acculturation in the international students' dietary practices while they are studying in England and how these differ from what they experienced in their home country. Moreover, this research also presents the contexts or factors that affect these phenomena.

Information about dietary practices in this study helps to illustrate the health and wellbeing problems that might arise when students are pursuing education overseas. Several issues that affected the international students' dietary experiences include time constraints and food preparation skills. Learners who plan to study overseas might benefit from equipping themselves in these areas; for example, receiving training on the importance of time management, as well as improving food preparation skills before leaving their home country.

Limited resources in this study highlighted the need for expanded research. Time and budget constraints affected the duration, sample size and data analysis methods used. Further quantitative studies with a large sample size can help to clarify if the experiences highlighted in our study are generalizable to a bigger population. While our study excluded participants who have been living in England for more than one year in order to avoid recall bias, longitudinal studies are required to report long-term dietary experiences along with any dietary changes, as well as factors affecting dietary practices that may emerge only after a certain period of time.

In the meantime, this study had enriched our understanding of what international students would encounter in terms of their dietary practices when studying abroad.

\section{REFERENCES}

Abraido-Lanza, A. F., Chao, M., \& Flórez, K. R. (2005). Do healthy behaviors decline with greater acculturation? Implications for the Latino mortality paradox. Social Science \& Medicine, 61(6), 12431255.

Alakaam, A. A., Castellanos, D. C., Bodzio, J., \& Harrison, L. (2015). The factors that influence dietary habits among international students in the United States. Journal of International Students, 5(2), 201-218.

Ansari, W.E., \& Stock, C. (2010). Is the health and wellbeing of university students associated with their academic performance? Cross sectional findings from the United Kingdom. International Journal of Environmental Research and Public Health, 7(2), 509-527. 
Ashakiran \& Deepthi, R. (2012). Fast foods and their impact on health. Journal of Krishna Institute of Medical Sciences University, 1(2), 715.

Brown, L., Edwards, J., \& Hartwell, H. (2010). A taste of the unfamiliar: Understanding the meanings attached to food by international postgraduate students in England. Appetite, 54, 202-207.

Cappellini, B., \& Yen, D. A. (2013). Little Emperors in the UK: Acculturation and food over time. Journal of Business Research, 66(8), 968-974.

Colaizzi, P. F. (1978). Psychological research as a phenomenologist views it. In R. Vaile \& M. King (Eds.), Existential Phenomenological Alternatives for Psychology (pp.48-71). New York, NY: Oxford University Press.

Coughlan, S. (2011). Record numbers of international students. BBC News. Retrieved from http://www.bbc.co.uk/news/business-12671198.

Edwards, J. S. A., Hartwell, H. L., \& Brown, L. (2010). Changes in food neophobia and dietary habits of international students. Journal of Human Nutrition and Dietetics, 23, 301-311.

Gilbert, P. A., \& Khokhar, S. (2008). Changing dietary habits of ethnic groups in Europe and implications for health. Nutrition Reviews, 66, 203-215.

Gordon-Larsen, P., Harris, K. M., Ward, D. S. \& Popkin, B. M. (2003). Acculturation and overweight-related behaviors among Hispanic immigrants to the US: The National Longitudinal Study of Adolescent Health. Social Science \& Medicine, 57(11), 2023-2034.

Hannigan, T. P. (2007). Homesickness and acculturation stress in the international student. In M. van Tilburg \& A. Vingerhoets (Eds.), Psychological Aspects of Geographical Moves: Homesickness and Acculturation Stress (pp.63-72). Amsterdam: Amsterdam University Press.

Hartwell, H. J., Edwards, J. S. A., \& Brown, L. (2011). Acculturation and food habits: Lessons to be learned. British Food Journal, 113(11), 1393-1405.

Higher Education Founding Council for England. (2014). Global demand for English higher education. Retrieved from http://www.hefce.ac.uk/media/hefce/content/heinengland/HEFCE20 14_08a.pdf.

Kremmyda, L., Papadaki, A., Hondros, G., Kapsokefalou, M., \& Scott, J.A. (2008). Differentiating between the effect of rapid dietary acculturation and the effect of living away from home for the first time, on the diets of Greek students studying in Glasgow. Appetite, 50, 455-463. 
Lara, M., Gamboa, C., Kahramanian, M. I., Morales, L. S., \& Bautista, D. E. H. (2005). Acculturation and Latino health in the United States: A review of the literature and its sociopolitical context. Annual Review of Public Health, 26(1), 367-397.

Matthews, A. E. (2007). Children and obesity: A pan-European project examining the role of food marketing. European Journal of Public Health, 18(1), 7-11.

McDonald, J. T., \& Kennedy, S. (2005). Is migration to Canada associated with unhealthy weight gain? Overweight and obesity among Canada's immigrants. Social Science \& Medicine, 61(12), 24692481.

Macrae, M. (1997). The induction of international students to academic life in the United Kingdom. In R. Harris \& D. McNamara (Eds.), Overseas Students in Higher Education: Issues in Teaching and Learning (pp.127-142). London: Routledge.

Morgan, J., \& Matthews, D. (2014). Home Office tightens rules on student visa refusals. Times Higher Education. Retrieved from http://www.timeshighereducation.co.uk/news/home-office-tightensrules-on-student-visa-refusals/2014854.article

Moustakas, C. (1994). Phenomenological research methods. Thousand Oaks, CA: Sage.

Pan, Y-L., Dixon, Z., Himburg, S., \& Huffman, F. (1999). Asian students change their eating patterns after living in the United States. Journal of the American Dietetic Association, 99, 54-57.

Papadaki, A., \& Scott, J. A. (2002). The impact of eating habits of temporary translocation from a Mediterranean to a Northern European environment. European Journal of Clinical Nutrition, 56, 455-461.

Perez-Cueto, F., Verbeke, W., Lachat, C., \& Remaut-De Winter, A. M. (2008). Changes in dietary habits following temporal migration. The case of international students in Belgium, Appetite, 52, 83-88.

Ramachandran, N. T. (2011). Enhancing international students' experiences: An imperative agenda for universities in the UK. Journal of Research in International Education, 10(2), 201-220.

Reeves, S. L., \& Henry, C. J. (2000). Dietary change, energy balance and body weight regulation among migrating students. International Journal of Food Sciences and Nutrition, 51, 429-438.

Ryan, J. (2011). Teaching and learning for international students: Towards a transcultural approach. Teachers and Teaching: Theory and Practice, 17(6), 631-648. 
Satia-Abouta, J. (2003). Dietary acculturation: Definition, process, assessment, and implications. International Journal of Human Ecology, 4(1), 71-86.

Shepherd, D. (1985). Dietary salt intake. Nutrition and Food Science, 85(5), $10-11$.

Shosha, G. A. (2012). Employment of Colaizzi's strategy in a descriptive phenomenology: A reflection of a researcher. European Scientific Journal, 8(27), 31-43.

Trichopoulou, A., Soukara, S., \& Vasilopoulou, E. (2007). Traditional foods: A science and society perspective. Trends in Food Science \& Technology, 18, 420-427.

Universities UK. (2014). The impact of universities on the UK economy. Retrieved from

http://www.universitiesuk.ac.uk/highereducation/Documents/2014/T heImpactOfUniversitiesOnTheUkEconomy.pdf.

Verbik, L., \& Lasanowski, V. (2007). International student mobility:

Patterns and trends. Retrieved from

http://www.eua.be/fileadmin/user_upload/files/newsletter/Internatio nal_Student_Mobility_-_Patterns_and_Trends.pdf.

Walsh, R. D. (1988). Husserl's epoche as method and truth. Auslegung, 14, 211-223.

NOVELLA O'SULLIVAN, MD, MSc, MA is an alumnus of Liverpool Hope University. She was a guest lecturer in Nutrition and research assistant in Education. Her current interest revolves around the many aspects of internationalization in higher education. Email: osullin@hope.ac.uk

FARZAD AMIRABDOLLAHIAN, BSc, PGCHE, PhD, FRSPH, FHEA, RNutr, is a Principal Lecturer and Subject Leader for Nutrition, teaching many aspects of the undergraduate curriculum and postgraduate research. $\mathrm{He}$ is an expert in zinc with his research into 'zinc adequacy of the UK population'. His other research interests are the 'dietary habits and health status of university students' and 'the assessment of energy expenditure'. He has a wide range of teaching experience, has published widely and is a Fellow of the Royal Society for Public Health, a Fellow of the Higher Education Academy and a Registered Nutritionist. Email: amirabf@hope.ac.u 